\title{
A family history of diabetes is not associated with arterial stiffness in non-diabetic Japanese population
}

\author{
Hirokazu Uemura ${ }^{1}$, Sakurako Katsuura-Kamano ${ }^{1}$, Miwa Yamaguchi ${ }^{1}$, Mariko Nakamoto ${ }^{2}$, \\ Mineyoshi Hiyoshi ${ }^{1}$, Fusakazu Sawachika ${ }^{1}$, Kokichi Arisawa ${ }^{1}$ \\ ${ }^{1}$ Department of Preventive Medicine, Institute of Health Biosciences, The University of Tokushima Graduate School, Tokushima, \\ Japan \\ ${ }^{2}$ Department of Public Health and Applied Nutrition, Institute of Health Biosciences, The University of Tokushima Graduate School, \\ Tokushima, Japan \\ Email: uemura@basic.med.tokushima-u.ac.jp
}

Received 9 August 2013; revised 9 September 2013; accepted 16 September 2013

Copyright (C) 2013 Hirokazu Uemura et al. This is an open access article distributed under the Creative Commons Attribution License, which permits unrestricted use, distribution, and reproduction in any medium, provided the original work is properly cited.

\section{ABSTRACT}

Prevalent diabetes is at high risk for cardiovascular diseases and has a high familial inheritance. However, little is known whether a non-diabetic subject with a family history of diabetes is at high risk for vascular damage or not. The purpose of this study was to evaluate the association between a family history of diabetes and arterial stiffness in adult non-diabetic Japanese population. We analyzed eligible 787 non-diabetic subjects (502 men and 285 women) aged 35 - 69 years who enrolled in the baseline survey of a cohort study in Tokushima Prefecture, Japan and who underwent a brachial-ankle pulse wave velocity (baPWV) measurement. Information on individual lifestyle characteristics including medical history and treatment for diseases and a first-degree family history of diabetes was obtained through a structured self-administered questionnaire. Analysis of covariance and logistic regression analyses were used to evaluate the association between a family history of diabetes and ba-PWV. We found no differences in age-and-systolic blood pressure-adjusted and multivariate-adjusted means of ba-PWVs between subjects of both sexes with and without a family history of diabetes. Logistic regression analyses including both sexes also revealed that subjects with a family history of diabetes showed no differences in age-and-systolic blood pressure-adjusted and multivariate-adjusted odds ratios for high ba-PWV compared to those without that trait. Our results suggest that a family history of diabetes itself is not associated with arterial stiffness in adult non-diabetic Japanese population.

Keywords: Arterial Stiffness; Brachial-Ankle Pulse
Wave Velocity; Family History of Diabetes; Cross-Sectional Study

\section{INTRODUCTION}

Type 2 diabetes has been globally increasing [1]. In Japan, the prevalence of diabetes was reported to be $11.9 \%$ in total ( $16.9 \%$ in men and $8.4 \%$ in women) in adults aged 40 - 74 years in 2011 [2], and it has rapidly increased during the past two decades [3]. Subjects with prevalent diabetes are at high risk for cardiovascular diseases [4,5], and cardiovascular events are global causes of death. It is important to assess the cardiovascular condition for preventing atherosclerosis in subjects who have risk factors of diabetes. Atherosclerotic changes in the arteries mainly contribute to the pathogenesis of cardiovascular disease, and increased arterial stiffness is associated with atherosclerosis [6]. Arterial stiffness can be assessed by measuring the pulse wave velocity (PWV) [7]. Carotid-femoral PWV (cf-PWV) is a noninvasive established assessment of aortic stiffness; its values are well correlated with vascular damage [8]. However, measuring cf-PWV is rather complicated and time consuming. Brachial-ankle PWV (ba-PWV) measurement is convenient, reproducible, and only requires a short time. Therefore, measuring ba-PWV has become popular in Asian countries for screening arterial atherosclerotic changes in large population.

Several risk factors have been determined for diabetes, and a family history is one of the recognized risk factors of diabetes [9]. However, little is known whether a nondiabetic subject with a family history of diabetes is at high risk for vascular damage or not. The purpose of this study was to evaluate the association between a family history of diabetes and arterial stiffness measured by ba- 
PWV in adult non-diabetic Japanese population.

\section{SUBJECTS AND METHODS}

\subsection{Study Subjects}

A total of 873 participants (573 men and 300 women) aged 35 - 69 years who enrolled in the baseline survey of a prospective cohort study in Tokushima Prefecture, Japan from November 2009 to January 2012 and who underwent ba-PWV measurement at the baseline survey were included in this cross-sectional study. The subjects were mostly office workers. This study was conducted as part of the prospective cohort study, that is named as the Japan Multi-Institutional Collaborative Cohort (J-MICC) Study, as described previously [10]. In brief, the aim of the J-MICC Study is to examine the associations of lifestyle and genetic factors and their interactions with lifestyle-related diseases. All participants in the J-MICC Study provided written informed consent. The ethics committees of Nagoya University School of Medicine (the affiliation of the former principal investigator [Nobuyuki Hamajima]), Aichi Cancer Center (the affiliation of the current principal investigator [Hideo Tanaka]), and the University of Tokushima Graduate School approved the protocol of this study.

\subsection{Questionnaire}

Individual lifestyle characteristics over the past year including medical history and treatment for diseases, smoking habit, alcohol drinking status, and leisure-time exercise were obtained through a structured self-administered questionnaire; trained staffs reviewed the accuracy of the mention contents to the questionnaire at the survey.

Leisure-time exercise was estimated based on the International Physical Activity Questionnaire (IPAQ) [11]. Exercise was divided into three levels: light exercise such as walking or hiking, moderate exercise such as light jogging or swimming, and vigorous exercise such as marathon running or competitive sports. The degrees of leisure-time exercise for the 3 levels were expressed as MET-hours per week (MET level $\mathrm{x}$ hours of activity $\mathrm{x}$ events per week) and summed [12]. In this estimation, 3.4 METs was assigned for light exercise, 7.0 METs for moderate exercise, and 10.0 METs for vigorous exercise.

Additionally, we obtained information regarding a firstdegree family history of diabetes (i.e., positive, negative, or unknown). When analyzed, a response of "positive" was regarded as having a family history of diabetes, whereas "negative" and "unknown" were regarded as not having it.

\subsection{Anthropometric and Biochemical Measurements}

Body height was obtained from the questionnaire and body weight was measured to the nearest $0.1 \mathrm{~kg}$ at the survey. Body mass index was calculated as weight (in $\mathrm{kg}$ ) divided by height (in $\mathrm{m}$ ) squared. Venous blood was aspirated from each participant and serum was separated within 3 hours. Serum lipid levels were measured at an external laboratory (BML Inc., Tokyo, Japan). Total cholesterol and triglycerides were determined by an enzyme assay, and high-density lipoprotein (HDL) cholesterol was determined by a direct method.

\subsection{PWV Measurement}

ba-PWV was measured using a waveform analyzer (model BP-203RPE III; Colin, Co. Ltd., Komaki, Japan) as described previously [13]. Briefly, the subject was examined while resting in the supine position in an air-conditioned room. Extremity blood pressure was measured using an oscillometric method, and the ankle-brachial pressure index (ABI) was automatically calculated. Heart rate was recorded simultaneously. ba-PWV was calculated by time-phase analysis between the right brachial artery pressure and volume waveforms at both ankles. To reduce inter-observer variation, all ba-PWV measurements were performed by a single researcher throughout the study. Individual ba-PWV and ABI data are expressed as the means of the bilateral ba-PWV and ABI, respectively.

\subsection{Statistical Analyses}

Among the 873 participants (573 men and 300 women) initially included in this cross-sectional study, we excluded 68 with a history of ischemic heart disease $(n=17)$, stroke $(\mathrm{n}=9)$ and diabetes $(\mathrm{n}=52)$. We excluded another 5 subjects who had a low right or left ABI (ABI $\leq$ 0.9 ), which suggested peripheral arterial occlusive disease and their ba-PWV values might be unreliable. After excluding an additional 13 subjects for whom serum lipid level data were missing, 787 non-diabetic subjects (502 men and 285 women) were included for analysis.

Continuous variables are expressed as mean \pm standard deviation, whereas those with skewed distribution are expressed as median (25 percentile, 75 percentile). Categorical variables are expressed as proportion (\%). Student's t-test, Wilcoxon rank sum test, or Fisher's exact test was used to compare the baseline characteristics between sexes and between subjects with and without a family history of diabetes. Prevalent hypertension was defined as systolic blood pressure $\geq 140 \mathrm{mmHg}$ or diastolic blood pressure $\geq 90 \mathrm{mmHg}$, or antihypertensive agent use. Hypercholesterolemia was defined as serum total cholesterol $\geq 220 \mathrm{mg} / \mathrm{dl}$ or receiving treatment for hypercholesterolemia, and low HDL cholesterol was as serum HDL cholesterol $<40 \mathrm{mg} / \mathrm{dl}$. Elevated triglycerides was defined as serum triglycerides $\geq 150 \mathrm{mg} / \mathrm{dl}$. 
We used analysis of covariance to evaluate the associations between a family history of diabetes and baPWV values, in men and women separately, after adjusting for the probable covariates. Adjusted covariates were as follows: 1) age (continuous) and systolic blood pressure $(<120,120$ to $<140,140$ to $<160$, or $\geq 160 \mathrm{mmHg}$ with no medical treatment, or anti-hypertensive agent use) (model 1), 2) age, systolic blood pressure, body mass index ( $\mathrm{kg} / \mathrm{m}^{2}$, quartiles), smoking habit (current, past, and never), alcohol drinking status (current, past, and never), leisure-time exercise (MET-hours/week, quartiles), hypercholesterolemia (no/yes), Low HDL cholesterol (no/yes), elevated triglycerides (no/yes), and heart rate (continuous) (model 2). Additionally, we evaluated the association between a family history of diabetes and high ba-PWV by logistic regression in all non-diabetic subjects adjusting for sex with the same covariates in the analysis of covariance. High ba-PWV was defined as a value exceeding the sex-specific median value $(1397$ and $1213 \mathrm{~cm} / \mathrm{s}$ in men and women, respectively). In the logistic regression analyses, categorical variables were converted into dummy variables, and these dummy variables except for reference categories were included in the model.

All calculations and statistical tests were performed using SAS, version 8.2 (SAS Institute Inc., Cary, NC, USA). All statistical tests were based on 2-sided probabilities, and $\mathrm{P}$ values $<0.05$ were considered statistically significant.

\section{RESULTS}

\subsection{Baseline Characteristics}

Age was significantly and positively correlated with baPWV in both sexes $(\mathrm{r}=0.458, \mathrm{P}<0.001$ in men, and $\mathrm{r}=$ $0.553, \mathrm{P}<0.001$ in women). Systolic blood pressure was also strongly and positively correlated with ba-PWV in both sexes $(\mathrm{r}=0.666, \mathrm{P}<0.001$ in men, and $\mathrm{r}=0.710, \mathrm{P}$ $<0.001$ in women).

Table 1 shows the baseline characteristics of the nondiabetic subjects by sex. Men showed significantly higher years of ages, body mass index, leisure-time exercise level, systolic and diastolic blood pressure, serum triglyceride level, and significantly lower serum HDL cholesterol level than women. Men also exhibited significantly higher rates of current smoking and drinking, and higher prevalence of hypertension, low HDL cholesterol, and elevated triglycerides than women. The ba-PWV values were considerably higher in men than in women. The prevalence rate of a family history of diabetes was not different between sexes.

Table 2 presents the baseline characteristics of the subjects with and without a family history of diabetes in non-diabetic subjects by sex. In men, although serum tri- glyceride level was significantly higher in subjects with a family history of diabetes than those without that trait, the other characteristics including ba-PWV values were not different between subjects with and without a family history of diabetes. In women, subjects with a family history of diabetes showed significantly lower prevalence of hypertension and low HDL cholesterol, and significantly lower systolic and diastolic blood pressure, and ba-PWV values.

\subsection{Association between a Family History of Diabetes and Arterial Stiffness in Non-Diabetic Subjects}

As shown in Table 3, there were no differences in ageand-systolic blood pressure-adjusted (model 1) and multivariate-adjusted (model 2) means of ba-PWVs between subjects of both sexes with and without a family history of diabetes. Logistic regression analyses including both sexes revealed that subjects with a family history of diabetes showed no differences in age-and-systolic blood pressure-adjusted (model 3) and multivariate-adjusted (model 4) odds ratios for high ba-PWV compared to those without that trait (Table 4).

\section{DISCUSSION}

The present study revealed that a family history of diabetes was not associated with arterial stiffness in both sexes in adult non-diabetic Japanese population.

cf-PWV is an established marker of aortic stiffness. Although ba-PWV, which can be measured more conveniently than cf-PWV, reflects the arterial stiffness of both large and mid-sized arteries [13,14], it is well correlated with cf-PWV as well as aortic PWV assessed using a direct catheter method [14]. In the current study, aging was strongly associated with increased ba-PWV in both sexes, and men had significantly higher ba-PWV values than women; these findings are concordant with other studies [13,15]. Aging affects structural and functional conditions within the arterial wall through the degeneration of elastic fibers and deterioration of endothelium-dependent vasodilation. Estrogen has been demonstrated to have protective effects against increased arterial stiffness [16]. In addition to age and gender, blood pressure is a well-recognized determinant of arterial stiffness [17]. Systolic blood pressure was strongly correlated with ba-PWV values in men and women $(r=0.666, \mathrm{P}<0.001$ in men and $\mathrm{r}=0.710, \mathrm{P}<0.001$ in women) in our subjects. Increased blood pressure and increased arterial stiffness may be associated with each other.

Diabetes has been increasing globally, and the number of people with diabetes has been estimated to increase from 153 million in 1980 to 347 million in 2008 [1]. Di- 
Table 1. Baseline characteristics of the non-diabetic subjects.

\begin{tabular}{|c|c|c|c|}
\hline & Men $($ No. $=502)$ & Women $($ No. $=285)$ & $P$ \\
\hline Age (years) ${ }^{a}$ & $48.4 \pm 8.5$ & $46.5 \pm 7.4$ & $<0.001$ \\
\hline Body mass index $\left(\mathrm{kg} / \mathrm{m}^{2}\right)^{\mathrm{a}}$ & $24.1(22.1,25.8)$ & $21.2(19.6,23.2)$ & $<0.001$ \\
\hline \multicolumn{4}{|l|}{ Smoking habit (\%) } \\
\hline Never & 30.7 & 88.4 & \\
\hline Past & 35.9 & 6.7 & $<0.001$ \\
\hline Current & 33.5 & 4.9 & \\
\hline \multicolumn{4}{|l|}{ Alcohol drinking status (\%) } \\
\hline Never & 24.7 & 56.5 & \\
\hline Past & 1.4 & 2.1 & $<0.001$ \\
\hline Current & 73.9 & 41.4 & \\
\hline Exercise (MET-hours/week) ${ }^{\mathrm{b}}$ & $4.25(1.28,15.30)$ & $2.55(0.00,8.93)$ & $<0.001$ \\
\hline Systolic BP $(\mathrm{mmHg})^{\mathrm{a}}$ & $134.0 \pm 17.0$ & $123.4 \pm 16.2$ & $<0.001$ \\
\hline Diastolic BP $(\mathrm{mmHg})^{\mathrm{a}}$ & $84.0 \pm 11.7$ & $74.7 \pm 10.9$ & $<0.001$ \\
\hline Heart rate $(\text { times } / \mathrm{min})^{\mathrm{a}}$ & $66.8 \pm 10.5$ & $67.9 \pm 8.7$ & 0.122 \\
\hline Total cholesterol $(\mathrm{mg} / \mathrm{dl})^{\mathrm{a}}$ & $213.0 \pm 32.8$ & $213.5 \pm 35.9$ & 0.821 \\
\hline HDL cholesterol $(\mathrm{mg} / \mathrm{dl})^{\mathrm{a}}$ & $56.5 \pm 11.9$ & $67.9 \pm 8.7$ & $<0.001$ \\
\hline Triglycerides $(\mathrm{mg} / \mathrm{dl})^{\mathrm{b}}$ & $113(79,165)$ & $68(51,96)$ & $<0.001$ \\
\hline \multicolumn{4}{|l|}{ Prevalence $(\%)$} \\
\hline Hypertension & 44.2 & 19.3 & $<0.001$ \\
\hline Hypercholesterolemia & 40.4 & 43.2 & 0.498 \\
\hline Low HDL cholesterol & 3.8 & 1.1 & 0.025 \\
\hline Elevated triglycerides & 30.9 & 9.8 & $<0.001$ \\
\hline Having a family history of diabetes (\%) & 23.3 & 27.0 & 0.264 \\
\hline $\mathrm{ABI}^{\mathrm{a}}$ & $1.12 \pm 0.06$ & $1.08 \pm 0.07$ & $<0.001$ \\
\hline Ba-PWV $(\mathrm{cm} / \mathrm{sec})^{\mathrm{a}}$ & $1424 \pm 235$ & $1245 \pm 202$ & $<0.001$ \\
\hline
\end{tabular}

${ }^{\mathrm{a}} \mathrm{Mean} \pm \mathrm{SD},{ }^{\mathrm{b}} \mathrm{Median}(25 \%, 75 \%)$; BP, blood pressure; HDL, high-density lipoprotein; ABI, ankle-brachial pressure index; ba-PWV, brachial-ankle pulse wave velocity.

abetes is associated with an increased risk of cardiovascular disease [4,5]. Atherosclerotic cardiovascular complication is one of the main factors of the high morbidity and mortality in diabetic subjects $[18,19]$. Arterial stiffness is demonstrated to increase in subjects with prevalent type 2 diabetes [20-22]. This finding was confirmed in the participants who enrolled in the baseline survey of our cohort study. The ba-PWV values in the excluded participants with history or treatment for diabetes were $1546 \pm 214 \mathrm{~cm} / \mathrm{s}$ in men and $1504 \pm 280 \mathrm{~cm} / \mathrm{s}$ in women, respectively, and these values were considerably higher than those in the non-diabetic subjects in this cross-sectional study $(1424 \pm 235 \mathrm{~cm} / \mathrm{s}$ in men and $1245 \pm 202$ $\mathrm{cm} / \mathrm{s}$ in women, as shown in Table 1). Subjects even at the condition before diabetic progression are recognized to be at high risk of cardiovascular disorder and mortality, and impaired fasting glucose has been reported to be associated with increased ba-PWV [23]. Increased arterial stiffness is considered to be one factor linking diabetes and cardiovascular complications.

Diabetes is well known to have a high familial inheritance, however, little is known whether a person who is not suffering from diabetes and having a family history of diabetes is at high risk for increased arterial stiffness or not. In the present study, we could not find an association between a family history of diabetes and arterial 
Table 2. Baseline characteristics of the subjects with and without a family history of diabetes in the non-diabetic subjects.

\begin{tabular}{|c|c|c|c|c|c|c|}
\hline & \multicolumn{3}{|c|}{ Men $($ No. $=502)$} & \multicolumn{3}{|c|}{ Women (No. = 285) } \\
\hline & \multicolumn{3}{|c|}{ Family history of diabetes } & \multicolumn{3}{|c|}{ Family history of diabetes } \\
\hline & No & Yes & $P$ & No & Yes & $P$ \\
\hline No. (\%) & $385(76.7)$ & $117(23.3)$ & & $208(73.0)$ & $77(27.0)$ & \\
\hline Age $(\text { years })^{\mathrm{a}}$ & $48.6 \pm 8.5$ & $47.8 \pm 8.3$ & 0.413 & $46.7 \pm 7.6$ & $45.9 \pm 6.8$ & 0.421 \\
\hline Body mass index $\left(\mathrm{kg} / \mathrm{m}^{2}\right)^{\mathrm{b}}$ & $24.1(22.0,25.8)$ & $24.3(22.8,26.1)$ & 0.291 & $21.1(19.6,23.0)$ & $21.5(20.0,23.5)$ & 0.430 \\
\hline \multicolumn{7}{|l|}{ Smoking habit (\%) } \\
\hline Never & 29.6 & 34.2 & & 88.5 & 88.3 & \\
\hline Past & 36.1 & 35.0 & 0.614 & 6.7 & 6.5 & 1.000 \\
\hline Current & 34.3 & 30.8 & & 4.8 & 5.2 & \\
\hline \multicolumn{7}{|l|}{ Alcohol drinking status (\%) } \\
\hline Never & 22.9 & 30.8 & & 52.9 & 66.2 & \\
\hline Past & 1.0 & 2.6 & 0.067 & 2.4 & 1.3 & 0.130 \\
\hline Current & 76.1 & 66.7 & & 44.7 & 32.5 & \\
\hline Exercise (MET-hours/week) ${ }^{b}$ & $4.25(1.28,15.30)$ & $4.25(0.43,14.18)$ & 0.718 & $2.55(0.43,8.90)$ & $2.55(0.00,12.23)$ & 0.722 \\
\hline Systolic BP $(\mathrm{mmHg})^{\mathrm{a}}$ & $133.6 \pm 17.2$ & $135.2 \pm 16.4$ & 0.378 & $124.8 \pm 16.5$ & $119.7 \pm 14.7$ & 0.018 \\
\hline Diastolic BP $(\mathrm{mmHg})^{\mathrm{a}}$ & $83.7 \pm 11.7$ & $84.7 \pm 11.6$ & 0.447 & $75.8 \pm 11.0$ & $71.8 \pm 10.2$ & 0.007 \\
\hline Heart rate (beats $/ \mathrm{min})^{\mathrm{a}}$ & $66.8 \pm 9.8$ & $66.8 \pm 12.4$ & 0.976 & $67.9 \pm 8.7$ & $67.8 \pm 8.7$ & 0.947 \\
\hline Total cholesterol $(\mathrm{mg} / \mathrm{dl})^{\mathrm{a}}$ & $212.3 \pm 32.6$ & $215.0 \pm 33.6$ & 0.438 & $215.7 \pm 35.0$ & $207.6 \pm 37.7$ & 0.090 \\
\hline HDL cholesterol $(\mathrm{mg} / \mathrm{dl})^{\mathrm{a}}$ & $57.1 \pm 12.0$ & $54.8 \pm 11.1$ & 0.068 & $70.1 \pm 13.1$ & $67.1 \pm 13.9$ & 0.088 \\
\hline Triglycerides $(\mathrm{mg} / \mathrm{dl})^{\mathrm{b}}$ & $108(77,156)$ & $128(86,184)$ & 0.013 & $70(52,99.5)$ & $64(49,95)$ & 0.481 \\
\hline \multicolumn{7}{|l|}{ Prevalence (\%) } \\
\hline Hypertension & 43.9 & 45.3 & 0.832 & 23.1 & 9.1 & 0.007 \\
\hline Hypercholesterolemia & 41.0 & 38.5 & 0.668 & 46.6 & 33.8 & 0.060 \\
\hline Low HDL cholesterol & 3.9 & 3.4 & 1.000 & 0.0 & 3.9 & 0.019 \\
\hline Elevated triglycerides & 28.8 & 37.6 & 0.086 & 9.6 & 10.4 & 0.825 \\
\hline $\mathrm{ABI}^{\mathrm{a}}$ & $1.12 \pm 0.06$ & $1.12 \pm 0.06$ & 0.294 & $1.08 \pm 0.07$ & $1.08 \pm 0.06$ & 0.692 \\
\hline $\mathrm{Ba}-\mathrm{PWV}(\mathrm{cm} / \mathrm{sec})^{\mathrm{a}}$ & $1425 \pm 232$ & $1421 \pm 247$ & 0.883 & $1261 \pm 204$ & $1201 \pm 192$ & 0.026 \\
\hline
\end{tabular}

${ }^{\mathrm{a}}$ Mean $\pm \mathrm{SD},{ }^{\mathrm{b}}$ Median $(25 \%, 75 \%)$; BP, blood pressure; HDL, high-density lipoprotein; ABI, ankle-brachial pressure index; ba-PWV, brachial-ankle pulse wave velocity.

stiffness after adjusting for known atherosclerotic risk factors in adult non-diabetic men and women. To verify the reliability of our results by analysis of covariance, we additionally conducted logistic regression analyses including both sexes to examine whether a family history of diabetes is associated with high ba-PWV defined as a value greater than its sex-specific median (i.e., $1424 \mathrm{~cm} / \mathrm{s}$ and $1245 \mathrm{~cm} / \mathrm{s}$ in men and women, respectively) in nondiabetic subjects, then no association was confirmed. This cut-off value for high ba-PWV may be suitable, because ba-PWV $>1400 \mathrm{~cm} / \mathrm{s}$ has been recommended for high ba-PWV due to its high sensitivity to predict cardiovascular diseases [24], and women have lower ba-PWV than men. If the cut-off value for high ba-PWV was replaced with a value greater than its sex-specific 75th percentile, the results were not altered. There were few reports on the association between a family history of diabetes and arterial stiffness. It was reported by Hopkins et al. [25] that a positive family history of type 2 diabetes $(n=22)$ was associated with decreased aortic distensibility in healthy young adult subjects $(\mathrm{n}=67)$, and this is not consistent with our finding. However, this study included small subjects and matched the subjects by only age and sex. It was recently reported by Li et al. [22] that impaired glucose tolerance and newly diagnosed diabetes had significantly higher ba-PWV values compared with 
Table 3. Association between a family history of diabetes and ba-PWV by analysis of covariance in the non-diabetic subjects.

\begin{tabular}{|c|c|c|c|c|c|}
\hline \multirow[b]{2}{*}{ No. $(\%)$} & \multicolumn{2}{|l|}{ Model 1} & \multicolumn{3}{|c|}{ Model 2} \\
\hline & $\begin{array}{c}\text { Adjusted } \\
\text { means }\end{array}$ & $P$ & $\begin{array}{c}\text { Adjusted } \\
\text { means }\end{array}$ & $\mathrm{SE}$ & $P$ \\
\hline
\end{tabular}

Family history of diabetes

$\begin{array}{lllllll}\text { no } & 385(76.7) & 1471.4 & 10.0 & 1496.2 & 28.9 \\ \text { yes } & 117(23.3) & 1459.4 & 16.6 & 1486.6 & 32.4 & 0.603\end{array}$

Women

Family history of diabetes

\begin{tabular}{cccccccc} 
no & $208(73.0)$ & 1347.6 & 12.8 & 1328.1 & 42.1 & \\
yes & $77(27.0)$ & 1325.1 & 17.9 & 0.198 & 1301.5 & 41.6 & \\
\hline
\end{tabular}

ba-PWV, brachial-ankle pulse wave velocity; SE, standard error; Model 1: adjusted for age and systolic blood pressure. Model 2: adjusted for age, systolic blood pressure, body mass index, smoking habit, alcohol drinking status, leisure-time exercise, hypercholesterolemia, low HDL cholesterol, elevated triglycerides, and heart rate.

Table 4. Odds ratios of a family history of diabetes for high baPWV in both sexes of the non-diabetic subjects.

\begin{tabular}{ccccccc}
\hline & & \multicolumn{2}{c}{ Model 3 } & \multicolumn{2}{c}{ Model 4 } \\
\cline { 3 - 7 } & No. (\%) & OR & $(95 \% \mathrm{CI})$ & OR & $(95 \% \mathrm{CI})$ \\
\hline \multicolumn{2}{c}{ Family history of diabetes } & & & & \\
no & $593(75.3)$ & 1 & & 1 & \\
yes & $194(24.7)$ & 0.81 & $(0.53-1.2)$ & 0.86 & $(0.55-1.4)$ \\
\hline
\end{tabular}

ba-PWV, brachial-ankle pulse wave velocity; OR, odd ratio; CI, confidence interval; Model 3: adjusted for age, sex, and systolic blood pressure. Model 4: adjusted for age, sex, systolic blood pressure, body mass index, smoking habit, alcohol drinking status, leisure-time exercise, hypercholesterolemia, low HDL cholesterol, elevated triglycerides, and heart rate.

normal glucose tolerance, however impaired fasting glucose did not have higher ba-PWV values after adjustment for age, sex, body mass index, waist to hip ratio, smoking, alcohol consumption, habitual exercise, systolic blood pressure, and lipid profiles. This study implies that pre-diabetic condition may not be associated with increased arterial stiffness after adjusted for the adequate covariates. The adjusted covariates in the study by Li et $a l$. were almost similar to those in the current study. Therefore, we consider that there is no surplus risk of the increased arterial stiffness in a subject who has a family history of diabetes but is not suffering from diabetes.

Our study has several limitations. First, information about the medical history or treatment for diseases including diabetes was self-reported; therefore, unrecognized cases may have existed. In addition, information about a family history of diabetes was also self-reported, therefore it might be underestimated. Second, our study included relatively few subjects, possibly lowering the statistical power. Finally, since all of our subjects were Japanese, these results may not be applicable to other ethnic populations.

\section{CONCLUSION}

In conclusion, the current study demonstrates that a family history of diabetes itself is not associated with arterial stiffness in adult non-diabetic Japanese population. Further large studies will be needed to confirm this conclusion.

\section{ACKNOWLEDGEMENTS}

This study was supported in part by Grants-in-Aid for Scientific Research on Priority Areas of Cancer (No. 17015018) and on Innovative Areas (No. 221S0001) from the Japanese Ministry of Education, Culture, Sports, Science and Technology. The authors declare that there is no conflict of interest.

\section{REFERENCES}

[1] Danaei, G., Finucane, M.M., Lu, Y., Singh, G.M., Cowan, M.J., Paciorek, C.J., Lin, J.K., Farzadfar, F., Khang, Y.H., Stevens, G.A., Rao, M., Ali, M.K., Riley, L.M., Robinson, C.A. and Ezzati, M.; Global Burden of Metabolic Risk Factors of Chronic Diseases Collaborating Group (Blood Glucose) (2011) National, regional, and global trends in fasting plasma glucose and diabetes prevalence since 1980: Systematic analysis of health examination surveys and epidemiological studies with 370 country-years and 2.7 million participants. Lancet, 378, 31-40. http://dx.doi.org/10.1016/S0140-6736(11)60679-X

[2] Ministry of Health, Labour and Welfare (Japan) (2011) National health and nutrition survey. http://www.mhlw.go.jp/bunya/kenkou/eiyou/h23-houkok u.html

[3] Iso, H. (2008) Changes in coronary heart disease risk among Japanese. Circulation, 118, 2725-2729. http://dx.doi.org/10.1161/CIRCULATIONAHA.107.7501 $\underline{17}$

[4] Fujishima, M., Kiyohara, Y., Kato, I., Ohmura, T., Iwamoto, H., Nakayama, K., Ohmori, S. and Yoshitake, T. (1996) Diabetes and cardiovascular disease in a prospective population survey in Japan: The Hisayama Study. Diabetes, 45, S14-S16. http://dx.doi.org/10.2337/diab.45.3.S14

[5] Asia Pacific Cohort Studies Collaboration. (2003) The effects of diabetes on the risks of major cardiovascular diseases and death in the Asia-Pacific region. Diabetes Care, 26, 360-366. http://dx.doi.org/10.2337/diacare.26.2.360

[6] Wada, T., Kodaira, K., Fujishiro, K., Maie, K., Tsukiyama, E., Fukumoto, T., Uchida, T. and Yamazaki, S. (1994) Correlation of ultrasound-measured common carotid ar- 
tery stiffness with pathological findings. Arteriosclerosis, Thrombosis, and Vascular Biology, 14, 479-482. http://dx.doi.org/10.1161/01.ATV.14.3.479

[7] Lehmann, E.D. (1999) Clinical value of aortic pulse-wave velocity measurement. Lancet, 354, 528-529. http://dx.doi.org/10.1016/S0140-6736(99)00179-8

[8] van Popele, N.M., Grobbee, D.E., Bots, M.L., Asmar, R., Topouchian, J., Reneman, R.S., Hoeks, A.P., van der Kuip, D.A., Hofman, A. and Witteman, J.C. (2001) Association between arterial stiffness and atherosclerosis: The Rotterdam study. Stroke, 32, 454-460. http://dx.doi.org/10.1161/01.STR.32.2.454

[9] Adeghate, E., Schattner, P. and Dunn, E. (2006) An update on the etiology and epidemiology of diabetes mellitus. Annals of the New York Academy of Sciences, 1084, 1-29. http://dx.doi.org/10.1196/annals.1372.029

[10] Hamajima, N. and J-MICC Study Group (2007) The Japan Multi-Institutional Collaborative Cohort Study (JMICC Study) to detect gene-environment interactions for cancer. Asian Pacific Journal of Cancer Prevention, 8, 317-323.

[11] Craig, C.L., Marshall, A.L., Sjöström, M., Bauman, A.E., Booth, M.L., Ainsworth, B.E., Pratt, M., Ekelund, U., Yngve, A., Sallis, J.F. and Oja, P. (2003) International physical activity questionnaire: 12-country reliability and validity. Medicine and Science in Sports and Exercise, 35, 1381-1395. http://dx.doi.org/10.1249/01.MSS.0000078924.61453.FB

[12] Ishikawa-Takata, K. and Tabata, I. (2007) Exercise and Physical Activity Reference for Health Promotion 2006 (EPAR2006). Journal of Epidemiology, 17, 177. http://dx.doi.org/10.2188/jea.17.177

[13] Tomiyama, H., Yamashina, A., Arai, T., Hirose, K., Koji, Y., Chikamori, T., Hori, S., Yamamoto, Y., Doba, N. and Hinohara, S. Influences of age and gender on results of noninvasive brachial-ankle pulse wave velocity measurement-A survey of 12,517 subjects. Atherosclerosis, 166, 303-309. http://dx.doi.org/10.1016/S0021-9150(02)00332-5

[14] Yamashina, A., Tomiyama, H., Takeda, K., Tsuda, H., Arai, T., Hirose, K., Koji, Y., Hori, S. and Yamamoto, Y. (2002) Validity, reproducibility, and clinical significance of noninvasive brachial-ankle pulse wave velocity measurement. Hypertension Research, 25, 359-364. http://dx.doi.org/10.1291/hypres.25.359

[15] Ahimastos, A.A., Formosa, M., Dart, A.M. and Kingwell, B.A. (2003) Gender differences in large artery stiffness pre- and post puberty. The Journal of Clinical Endocrinology and Metabolism, 88, 5375-5380.

http://dx.doi.org/10.1210/jc.2003-030722

[16] Rajkumar, C., Kingwell, B.A., Cameron, J.D., Waddell, T., Mehra, R., Christophidis, N., Komesaroff, P.A., McGrath, B., Jennings, G.L., Sudhir, K. and Dart, A.M. (1997) Hormonal therapy increases arterial compliance in postmenopausal women. Journal of the American College of Cardiology, 30, 350-356.

\section{http://dx.doi.org/10.1016/S0735-1097(97)00191-5}

[17] Scuteri, A., Orru', M., Morrell, C.H., Tarasov, K., Schlessinger, K.D., Uda, M. and Lakatta, E.G. (2012) Associations of large artery structure and function with adiposity: Effects of age, gender, and hypertension. The SardiNIA Study. Atherosclerosis, 221, 189-197.

http://dx.doi.org/10.1016/j.atherosclerosis.2011.11.045

[18] Haffner, S.M., Lehto, S., Rönnemaa, T., Pyörälä, K. and Laakso, M. (1998) Mortality from coronary heart disease in subjects with type 2 diabetes and in nondiabetic subjects with and without prior myocardial infarction. The New England Journal of Medicine, 339, 229-234. http://dx.doi.org/10.1056/NEJM199807233390404

[19] Clarke, P.M., Gray, A.M., Briggs, A., Farmer, A.J., Fenn, P., Stevens, R.J., Matthews, D.R., Stratton, I.M. and Holman, R.R. (2004) A model to estimate the lifetime health outcomes of patients with type 2 diabetes: The United Kingdom Prospective Diabetes Study (UKPDS) Outcomes Model (UKPDS no. 68). Diabetologia, 47, 17471759. http://dx.doi.org/10.1007/s00125-004-1527-Z

[20] Suzuki, E., Kashiwagi, A., Nishio, Y., Egawa, K., Shimizu, S., Maegawa, H., Haneda, M., Yasuda, H., Morikawa, S., Inubushi, T. and Kikkawa, R. (2001) Increased arterial wall stiffness limits flow volume in the lower extremities in type 2 diabetic patients. Diabetes Care, 24, 2107-2114. http://dx.doi.org/10.2337/diacare.24.12.2107

[21] Kimoto, E., Shoji, T., Shinohara, K., Inaba, M., Okuno, Y., Miki, T., Koyama, H., Emoto, M. and Nishizawa, Y. (2003) Preferential stiffening of central over peripheral arteries in type 2 diabetes. Diabetes, 52, 448-452. http://dx.doi.org/10.2337/diabetes.52.2.448

[22] Li, C.H., Wu, J.S., Yang, Y.C., Shih, C.C., Lu, F.H. and Chang, C.J. (2012) Increased Arterial Stiffness in Subjects with Impaired Glucose Tolerance and Newly Diagnosed Diabetes but Not Isolated Impaired Fasting Glucose. The Journal of Clinical Endocrinology and Metabolism, 97, E658-E662. http://dx.doi.org/10.1210/jc.2011-2595

[23] Cruickshank, K., Riste, L., Anderson, S.G., Wright, J.S., Dunn, G. and Gosling, R.G. (2002) Aortic pulse-wave velocity and its relationship to mortality in diabetes and glucose intolerance: an integrated index of vascular function? Circulation, 106, 2085-2090.

http://dx.doi.org/10.1161/01.CIR.0000033824.02722.F7

[24] Yamashina, A., Tomiyama, H., Arai, T., Hirose, K., Koji, Y., Hirayama, Y., Yamamoto, Y. and Hori, S. (2003) Brachial-ankle pulse wave velocity as a marker of atherosclerotic vascular damage and cardiovascular risk. Hypertension Research, 26, 615-622. http://dx.doi.org/10.1291/hypres.26.615

[25] Hopkins, K.D., Lehmann, E.D., Jones, R.L., Turay, R.C. and Gosling, R.G. (1996) A family history of NIDDM is associated with decreased aortic distensibility in normal healthy young adult subjects. Diabetes Care, 19, 501-503. http://dx.doi.org/10.2337/diacare.19.5.501 